\title{
Myeloproliferative neoplasms in children
}

\author{
Inga Hofmann ${ }^{1,2}$
}

Received: 20 July 2015 / Accepted: 22 July 2015 /Published online: 2 August 2015

(C) Springer-Verlag Berlin Heidelberg 2015

\begin{abstract}
Myeloproliferative neoplasms (MPNs) are a group of clonal hematopoietic stem cell disorders characterized by aberrant proliferation of one or more myeloid lineages often with increased immature cells in the peripheral blood. The three classical BCR-ABL-negative MPNs are (1) polycythemia vera (PV), (2) essential thrombocythemia (ET), and (3) primary myelofibrosis (PMF), which are typically disorders of older adults and are exceedingly rare in children. The diagnostic criteria for MPNs remain largely defined by clinical, laboratory, and histopathology assessments in adults, but they have been applied to the pediatric population. The discovery of the $J A K 2$ V617F mutation and, more recently, MPL and calreticulin $(C A L R)$ mutations are major landmarks in the understanding of MPNs. Nevertheless, they rarely occur in children, posing a significant diagnostic challenge given the lack of an objective clonal marker. Therefore, in pediatric patients, the diagnosis must rely heavily on clinical and laboratory factors and exclusion of secondary disorders to make an accurate diagnosis of MPN. This review focuses on the clinical presentation, diagnostic work up, differential diagnosis, treatment, and prognosis of the classical BCR-ABL-negative MPNs (PV, ET, and PMF) in children and highlights the key differences to the adult diseases. Particular attention will be given to pediatric PMF, as it is the only disorder of this group that is observed
\end{abstract}

Inga Hofmann

inga.hofmann@childrens.harvard.edu

1 Pediatric Hematology/Oncology, Dana-Farber/Boston Children's Cancer and Blood Disorders Center Boston, 300 Longwood Ave, Bader 127.1, Boston, MA 02115, USA

2 Department of Pathology, Boston Children's Hospital, Harvard Medical School, Boston, MA, USA in infants and young children, and in many ways appears to be a unique entity compared to adult PMF.

Keywords Myeloproliferative neoplasms · Pediatric myeloproliferative neoplasms · Primary myelofibrosis · Essential thrombocythemia · Polycythemia vera

\section{Introduction}

The 2008 World Health Organization (WHO) Classification of Tumours of Haematopoietic and Lymphoid Tissues defines myeloproliferative neoplasms (MPNs) as a phenotypically diverse group of clonal hematopoietic stem cell (HSC) disorders characterized by (1) hyperproliferation of one or more of the components of the myeloid compartment and (2) evidence of increased immature cellular forms in the peripheral blood (PB) [72].

These conditions were first described in 1951 by William Damashek as "myeloproliferative disorders" (MPD), a group which included four distinct clinico-pathologic entities: (1) chronic myelogenous leukemia (CML), (2) polycythemia vera (PV), (3) essential thrombocythemia (ET), and (4) primary myelofibrosis (PMF). These "classical MPDs" were originally categorized based on the abnormal proliferation of a predominant myeloid compartment (i.e., granulocytic in CML, erythroid in PV, megakaryocytic in ET).

In 2008, the WHO revised the classification for MPDs and renamed those disorders myeloproliferative neoplasms (MPNs) to emphasize their clonal and therefore malignant nature. Under the WHO classification, MPNs now include the four "classic MPDs" (1) PV, (2) ET, (3) PMF, and (4) CML as well as the following conditions: (1) chronic neutrophilic leukemia (CNL), (2) chronic eosinophilic leukemia, not otherwise specified (CEL, NOS), (3) systemic mastocytosis, 
and (4) myeloproliferative neoplasm (MPN), unclassifiable. Finally, myeloid and lymphoid neoplasms associated with eosinophilia and abnormalities of platelet-derived growth factor receptor $\alpha$ and $\beta$ (PDGFRA and PDGFRB), or fibroblast growth factor receptor 1 (FGFR1) were also incorporated into the WHO classification as a new subcategory.

While the diagnostic criteria for MPNs remain largely defined by clinical, laboratory, and histopathology assessments, advances in genetics have identified abnormalities in receptor tyrosine kinases (TKs) in a large subset of patients. The identification of genetic alterations in TKs $(J A K 2, M P L)$ and recently calreticulin $(C A L R)$ has advanced our understanding of MPNs and will likely shape future classification and diagnostic strategies.

With the exception of CML, which is Philadelphia-positive $(\mathrm{Ph}+)$, the remaining classical MPNs (PV, ET, and PMF) present as Philadelphia-negative $\left(\mathrm{Ph}^{-}\right)$conditions primarily occurring in middle and advanced age adult patients [34]. Apart from CML, these disorders, particularly PMF and ET, are exceptionally rare in children and adolescents [27], with an incidence approximately $100 \times$ less than that of adults [79]. PV has only been reported anecdotally in pediatrics [25] and is generally observed in teenagers or young adults. Other pediatric disorders with features of MPNs, such as myelodysplastic syndromes/ myeloproliferative neoplasms (MDS/MPNs), particularly juvenile myelomonocytic leukemia (JMML), are unique disorders of childhood and are discussed separately in this issue in a report by Calvo et al.

Given the rarity of these conditions and challenges in the diagnosis of MPNs in pediatric patients, this review focuses on PV, ET, and PMF. Their clinical presentation, diagnostic work up, differential diagnosis, treatment, and prognosis in pediatric patients are discussed. Among the classical MPNs, only PMF has been observed in young children and infancy and appears to be a unique disorder different from adult PMF, which is also discussed in greater detail in this review.

Historically, the clinical and hematologic findings in children with $\mathrm{Ph}-\mathrm{MPN}$ were considered similar to those in adults. As a result, diagnostic criteria developed for adult patients with $\mathrm{Ph}-\mathrm{MPN}$ and were subsequently applied to pediatric cases $[50,77,86]$. Likewise, treatment approaches have been adopted on the basis of adult recommendations [13, 22, $25,38,44]$. More recently, however, several studies have identified important differences between adult and pediatric patients with PV, ET, and PMF. For example, the majority of adult patients with $\mathrm{Ph}-\mathrm{MPN}$ are due to the JAK2 V617F; this variant occurs at a much lower frequency in children with MPNs $[59,80]$. Although the pediatric literature on MPN is sparse, there appear to be critical differences in the differential diagnosis, natural history, clinical outcomes, and treatment approaches when compared to adult patients with MPN [22, $25,59,80,82]$. Therefore, a specific pediatric approach to $\mathrm{Ph}-$ MPN (PV, ET, and PMF) in children and young adults should be considered and is discussed in detail in this review.

\section{Polycythemia vera}

\section{Definitions and epidemiology}

PV is defined as an elevated red cell mass (RCM) after secondary conditions of erythrocytosis, such as hypoxia or inappropriate erythropoietin (EPO) production, have been ruled out. The bone marrow (BM) in PV is notable for massively increased erythropoiesis. A concurrent increase in platelets and granulocytes in the PB may be also noted. PV is the most common MPN in adults with an incidence of 1.1 cases per 100,000 per year. It is typically a disorder of older individuals with a median age at diagnosis of 60-65 years.

Designated studies on pediatric PV are limited to small case series and case reports. Therefore, the exact incidence of PV in pediatric patients is unknown but is estimated to be approximately 2 cases per 10 million persons under 20 years old per year [45]. Similar to PV in adults, there appears to be a male predominance at a ratio of approximately $2: 1[25,80]$. The median age at diagnosis in pediatric PV is 16 years [25].

\section{Pathophysiology and molecular genetics}

PV was among the first hematopoietic conditions demonstrated to be a clonal disorder. All PV cells of myeloid origin (as well as subpopulations of lymphoid cells) originate from the malignant clone, supporting the theory that PV arises from a pluripotent HSC.

Almost all adult patients (90-95\%) with PV carry a somatic activating mutation, $\mathrm{V} 617 \mathrm{~F}$ in the TK JAK2. JAK2 is an intracellular signaling molecule that is coupled to hematopoietic growth factor receptors, including EPO and thrombopoietin (TPO). JAK2 V617F causes activation of the $J A K 2$ kinase domain leading to EPO-independent autonomous erythropoiesis. Most of the remaining JAK2 V617Fnegative PV cases have activating mutations in exon 12 of $J A K 2$ and, unlike most cases of $\mathrm{PV}$, are not associated with thrombocytosis and leukocytosis. The prevalence of JAK2 $V 617 F$ mutations is much lower in pediatric PV compared to adults [25, 32], suggesting an age-dependent increase of acquired somatic mutation in $J A K 2$. Interestingly, there have been case reports of prenatal origin of $J A K 2 \mathrm{~V} 617 \mathrm{~F}$ mutations in young PV patients [35].

\section{Clinical features}

In adults, approximately $80 \%$ of PV cases are symptomatic at the time of diagnosis. The majority of patients present with headache, pruritus, and fatigue. Other common signs include dyspnea, dizziness, visual changes, weight loss, epigastric pain, paresthesias (erythromelalgia of hands and feet), and excessive sweating, all of which are thought to be secondary to hyperviscosity from increased RCM [8]. Only about half of 
pediatric PV patients present with symptoms related to polycythemia [25]. Thrombosis and bleeding are significant causes of morbidity and death in adult patients with PV occurring in $\sim 30$ and $8 \%$ respectively $[8,43]$. In contrast, no pediatric PV patients experienced these complications after a median follow-up of $>9$ years $[8,25]$.

On clinical exam, common findings in adults include hepatosplenomegaly, plethora, cutaneous ulcers, and pulmonary hypertension; however, these findings are less common in children. Splenomegaly is seen in about one third of pediatric PV patients $[8,25]$.

\section{Differential diagnosis}

In order to establish the diagnosis of PV, it is important to distinguish between absolute polycythemia - defined by an increase in the total RCM and present in $\mathrm{PV}$ - and relative polycythemia - defined by a decreased plasma volume and found secondary to intravascular hypovolemia. Traditionally, isotope testing was used to make an exact determination of the absolute RCM. However, this has become less important with the discovery of JAK2 mutations, which can by used to confirm a molecular diagnosis.

In addition, it is important to differentiate PV from secondary causes of erythrocytosis, usually related to elevated EPO levels. Appropriately high EPO levels may occur in setting of chronic hypoxia (e.g., high altitude, sleep apnea, smoking), while inappropriately high EPO levels may occur with exogenous EPO administration (e.g., "blood doping," chronic kidney disease) or endogenous EPO overproduction (e.g., renal, liver or other tumors, renal transplantation) [57]. Specific causes of secondary polycythemia in neonates and children include increased $\mathrm{Hgb}$ in the neonatal period, twin-twin transfusion syndrome or maternal-fetal bleeds, polycythemia of the newborn (common in infants born to diabetic mothers), adrenal hyperplasia, thyrotoxicosis, trisomy 13,18 , or 21 , high oxygen affinity hemoglobin, and congenital low 2,3bisphosphoglycerate. Furthermore, congenital causes of erythrocytosis (familial polycythemic states) need to be ruled out to establish the diagnosis of PV, which include (1)
Chuvash-type polycythemia due to $V H L$ mutations leading to autonomous high EPO production and (2) autosomal dominant polycythemia due to truncating EPO receptor mutations $[12,57]$.

\section{Laboratory and histopathology features}

In pediatric $\mathrm{PV}$, features on laboratory testing are similar to those found in adults and include (1) leukocytosis with a mean WBC of $6.7-10.8 \times 10^{9} / \mathrm{L},(2)$ erythrocytosis with a hemoglobin level (Hgb) of $18.0 \mathrm{~g} / \mathrm{dL}$ and hematocrit (Hct) of 53.2 $55 \%$ and (3) platelet count of $207-394 \times 10^{9} / \mathrm{L}[25,80]$. Other common laboratory features include elevated LDH and hyperuricemia.

The BM in pediatric PV is typically hypercellular with erythroid hyperplasia. Increased megakaryocytes, including large atypical forms with hyperlobated nuclei, are often noted. Mildly increased reticulin can be seen in some patients.

The revised WHO diagnostic criteria for PV are outlined in Table 1 and require the presence of a $J A K 2$ mutation and an increased RCM (major criteria). In contrast to adults, JAK2 $V 617 F$ mutations occur only in about $30 \%$ of sporadic pediatric PV patients and are not seen in hereditary erythrocytosis. Thus, the major diagnostic criteria cannot be fulfilled in most pediatric patients and two minor criteria need to be met to make a formal diagnosis [76]. Based on a number of smaller case series, all pediatric patients present with a hypercelullar marrow, but only $30 \%$ have a low serum EPO level or increase endogenous erythroid colony (EEC) growth. Therefore, the current diagnostic criteria for PV are suboptimal in children as the diagnosis can only be confirmed in approximately $50 \%$ of pediatric patients [81].

\section{Treatment, clinical outcome, and prognosis}

$\mathrm{PV}$ is a chronic disorder for which hematopoietic stem cell transplant (HSCT) is the only curative therapy. Given the high morbidity and mortality associated with HSCT and the generally favorable prognosis with chronic indolent disease, HSCT has been reserved for younger patients with poor prognosis. Studies

Table 1 Revised diagnostic criteria for polycythemia vera

\begin{tabular}{ll}
\hline Major criteria & Minor criteria \\
\hline $\begin{array}{l}\text { All criteria must be met }{ }^{\mathrm{a}} \\
\begin{array}{l}\text { 1. } \mathrm{Hgb}>18.5 \mathrm{~g} / \mathrm{dL} \text { in men, }>16.5 \mathrm{~g} / \mathrm{dL} \text { in women, or other evidence of } \\
\text { increased red cell volume }\end{array}\end{array}$ & $\begin{array}{l}\text { At least } 1 \text { criterion must be met }^{\mathrm{a}} \\
\text { 1. Hypercellular BM biopsy for age with trilineage hematopoiesis } \\
\text { (panmyelosis) with prominent erythroid, granulocytic, and } \\
\text { megakaryocytic proliferation } \\
\text { 2. JAK2 V617F or functional similar JAK2 mutation (such as exon 12) }\end{array}$ \\
& $\begin{array}{l}\text { 2. Serum erythropoietin level below the reference range for normal } \\
\text { 3EC growth formation in vitro }\end{array}$
\end{tabular}

${ }^{\mathrm{a}}$ If only the first major criterion is met, at least 2 of the minor criteria must be met to establish a diagnosis of polycythemia vera $E E C$ endogenous erythroid colony 
on HSCT outcomes of children with PV do not exist and only a single successful case report has been described [60]. Pediatric treatment approaches and outcomes have recently been reported in the largest pediatric case series ever, which included longterm follow-up to 10 years [25]. In this series, a majority of children with PV were managed with phlebotomy or erythrocytapheresis $(75 \%)$. A subset of patients required additional hydroxyurea (HU) $(25 \%)$ to reduce progressive splenomegaly and decrease Hct. Antiplatelet therapy with low-dose aspirin has also been utilized. In contrast to adults, who are at increased risk for marrow fibrosis, leukemia (25-50\% of postpolycythemic patients with marrow fibrosis develop acute myeloid leukemia (AML)), and thrombohemorrhagic events, none of these events occurred in children after a median follow-up time of 9.4 years. Only two patients (18\%) developed splenomegaly with associated grade 2 marrow fibrosis at 3 and 7 years, respectively [25]. The long-term life expectancy for pediatric PV patients is unknown.

\section{Essential thrombocythemia}

\section{Definitions and epidemiology}

Essential thrombocythemia (ET) is characterized by increased megakaryopoiesis, persistent thrombocytosis, the risk of vascular complications (including thrombosis and bleeding), and the risk of leukemic transformation $[14,77]$. ET is the second most common MPN in adults with a median age at diagnosis of 60 years and an incidence of $0.5-2$ cases per 100,000 persons per year $[16,24,26,47,55,90]$. There is a female-tomale predominance of 1.5-2:1, which is more skewed towards female patients in younger populations. Epidemiologic studies on pediatric ET are rare. The incidence of pediatric ET is estimated at 1 case in 10,000,000 children $(<14$ years old) per year [27] with a median age at diagnosis of 6.5 to 17 years and a wide age distribution (range from 0.2 to 19 years) $[23,37]$. Similar to adult ET patients, there is a female predominance [25]. ET is an acquired condition and must be distinguished from hereditary or familial forms of thrombocythemia. In these disorders, which are generally autosomal dominant, thrombocythemia occurs at a younger age and has been described in infants [25].

\section{Pathophysiology and molecular genetics}

Almost all patients with ET (90-95 \%) have a normal karyotype. JAK2 V617F mutations are present in 40-50\% of adult patients and are associated with higher Hgb levels and neutrophil counts. A small subset of adult ET patients (3-5\%) has an acquired somatic activating mutation in the TPO receptor $(M P L)$. Recently, mutations in CALR have also been identified on double negative $\left(J A K 2^{-}, M P L^{-}\right)$cases of ET. Germline mutations in $M P L$ [19], as well as rare cases of non-JAK2 $V 617 F$ (variants V617I and R564Q), have been found in families with hereditary thrombocytosis [46]. In addition, next generation sequencing (NGS) studies have identified somatic mutations on ASXL1 and TET2 and might be suggestive of clonal evolution [11, 18, 75].

Similar to other MPNs, ET shows increased in vitro sensitivity to cytokines with increased colony formation (i.e., EEC growth), megakaryocytic progenitor sensitivity to TPO, and endogenous megakaryocyte colony formation [20, 49].

\section{Clinical features}

The majority of children with ET are asymptomatic (65$75 \%$ ), and thrombocytosis is discovered as an incidental finding on routine evaluation. If symptoms occur, they are often non-specific with headaches being the most common in children [25]. Splenomegaly is found in up to $19 \%$ of pediatric patients and hepatomegaly can be seen on occasion [25]. The majority of reported symptoms are vasomotor in nature and are thought to arise due to abnormal platelet-endothelial interactions. Inappropriate vasomotor response leads to circulatory problems that manifest as neurological symptoms such as dizziness, headaches, syncope, transient ischemic attacks, acroparesthesias, and vision changes. Splenomegaly is present in about $25 \%$ of adult patients. Other symptoms may include ischemia, nausea, vomiting, and abdominal pain. In contrast to adults who have a 10-25\% incidence of thromboembolic events at diagnosis, children almost never present with thrombosis [25]. A small subset of adult ET patients (4\%) can suffer from bleeding complications, which have not been reported in pediatric case series. However, extreme thrombocytosis $\left(>1000 \times 10^{9} / \mathrm{L}\right)$ can lead to acquired von Willebrand disease (vWD) in some cases.

\section{Differential diagnosis}

The diagnosis of ET requires a persistent thrombocytosis, currently defined as a platelet count of $>450 \times 10^{9} / \mathrm{L}$, as well as requires the exclusion of alternative diagnosis including (1) reactive thrombocytosis, (2) other disorders that lead to thrombocytosis such as other MPNs, and (3) specific subclasses of MDS (eg., 5q- syndrome, refractory anemia with ringed sideroblasts associated with marked thrombocytosis (RARS-T)), the latter being extremely rare in children. Common causes of reactive thrombocytosis in children are outlined in Table 2 .

\section{Laboratory features and histopathology}

Similar to adult ET, pediatric ET patients show moderate leukocytosis with a mean WBC of $8.4-9.8 \times 10^{9} / \mathrm{L}$. The mean Hct is $\sim 39.8 \%$ and is higher in patients with sporadic forms of ET 
Table 2 Causes of reactive (secondary) thrombocytosis in children

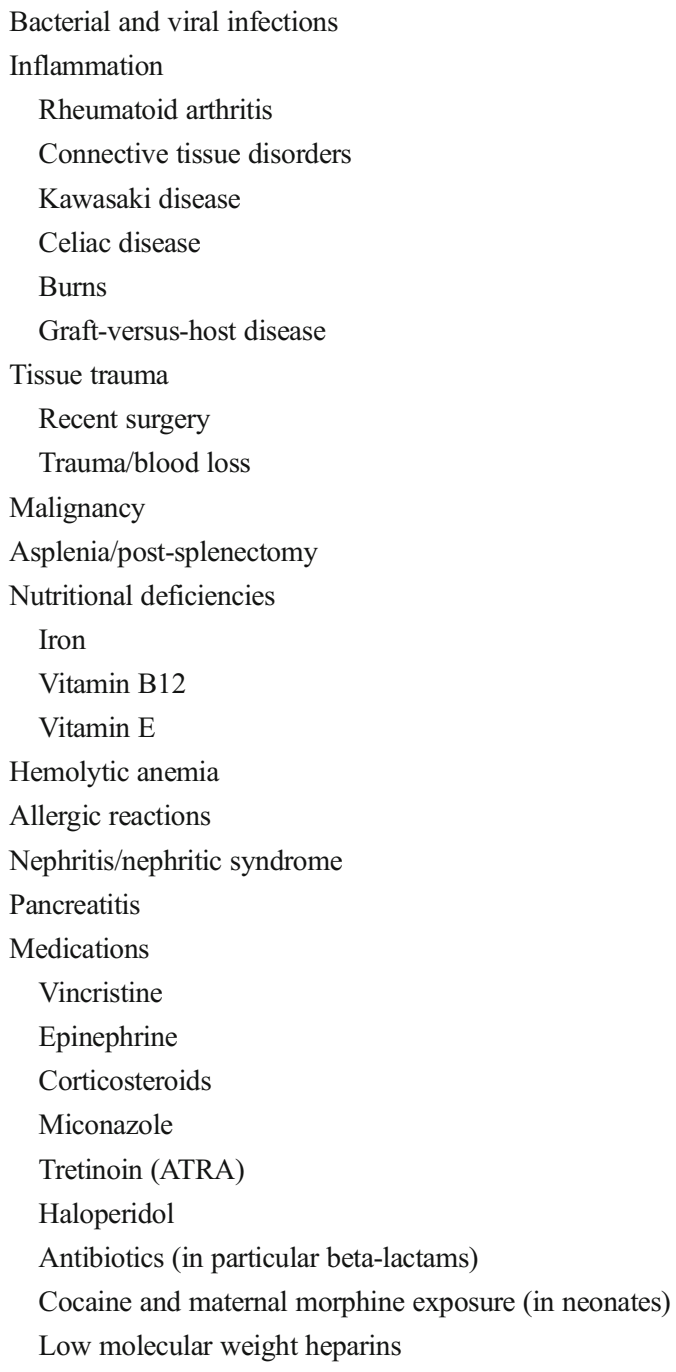

Adapted from [37]

than in hereditary forms. Thrombocytosis - the hallmark of ET - is typically observed at a mean value of $1109 \times 10^{9} / \mathrm{L}$ [25]. The PB smear often shows anisocytosis of the platelets with large to giant platelets and occasional circulating megakaryocyte fragments. White and red blood cell morphology is usually unremarkable. If significant leukoerythroblastosis and teardrop cells are present, a diagnosis of PMF or pre-fibrotic stage of PMF should be considered. Howell-Jolly bodies can be seen in functional hyposplenism or post-splenectomy patients.

The BM histology in pediatric ET is similar to those in adults and is characterized by a normocellular to hypercellular marrow. BM histology may also demonstrate increased megakaryocytes occurring in clusters with large megakaryocytes (Fig. 1). Megakaryocytes show abundant cytoplasm and prominent nuclear lobation (hyperploid nuclei) and often "staghorn-like" megakaryocytes. Occasional "cloud-like" forms traditionally thought to be more characteristic of PMF can be seen in some ET cases as well [89]. Reticulin fibrosis is absent or low-grade $(0-1 / 1)$ typically; low-grade fibrosis is seen in $20 \%$ of pediatric patients. If fibrosis is more pronounced, the diagnosis if PMF should be considered. The factors highlight that the differential diagnosis of true ET from prefibrotic myelofibrosis based on histologic criteria described in the WHO remains challenging [89] (Fig. 2). Ultimately novel molecular makers such as CALR might be more helpful to diagnosis and influence future classification systems.

Dysplasia is minimal or absent and, if present, MDS with fibrosis should be considered. Ringed sideroblasts are usually not seen and if noted in $>15 \%$ of erythroid precursors, the rare provisional entity of RARS-T should be contemplated. The current WHO diagnostic criteria for ET are outlined in Table 3.

Similar to adult ET, JAK2 V617F mutations are present in $40-50 \%$ of sporadic pediatric cases but are not found in hereditary forms of pediatric ET [25]. Clonal hematopoiesis can be demonstrated in the majority of female pediatric patients with sporadic ET. In contrast, the recent study by Giona et al. found germline-activating MPL S505A mutations in $94 \%$ of hereditary ET cases [25].

\section{Treatment options and prognosis}

Treatment options for pediatric ET are largely adopted from adult populations and are widely heterogeneous. Treatment for adult ET is typically administered after a risk stratification system has been applied. High-risk patients are usually defined as older ( $>60$ years) with prior history of thromboembolic events. Low-risk patients are younger ( $<60$ years) without history of thrombosis or cardiovascular risk factors. A standard treatment approach for pediatric ET does currently not exist. However, therapeutic approaches usually center on antiplatelet or cytoreductive therapy. Most pediatric patients are started on low-dose aspirin, which is generally recommended for low-risk adult ET patients as well. An important exception to the use of low-dose aspirin is in patients with extremely high platelet count as they are at risk for acquired vWD, which should be ruled out by ristocetin co-factor activity prior to initiating therapy. Cytoreductive therapy is indicated in high-risk adult ET patients and has been used in $50 \%$ of pediatric ET patients, primarily in sporadic ET cases [25]. The most common agents used are HU and anagrelide (ANA) followed by single interferon- $\alpha$ (IFN- $\alpha)$ or in combination with HU [25].

Recently, clinical trials of targeted JAK2 inhibitors, such as ruxolitinib, have shown encouraging results in adult patients. Several pediatric investigators have proposed risk adaptive treatment strategies for pediatric ET patients that may be considered [37]. 


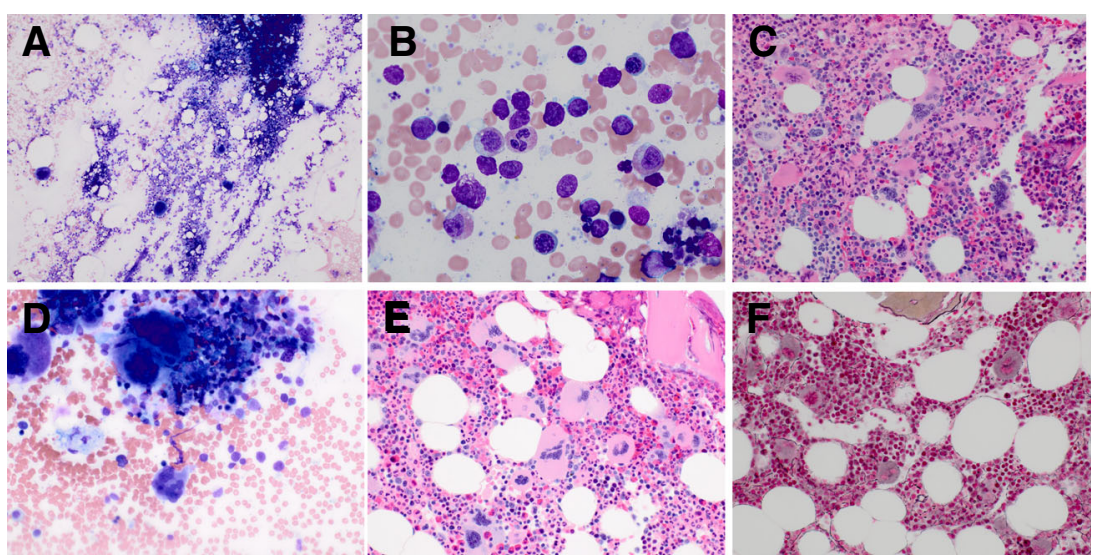

Fig. 1 Histopathology of pediatric essential thrombocythemia (ET). a-c Representative images from the marrow of an 18-year-old female with marked thrombocytosis of $1200 \times 10^{9}$ cells/L. The hemoglobin was normal. No organomegaly was present. The BM karyotype was normal. Molecular studies confirmed a JAK2 V617F mutation. Given the clinical and histopathologic features, the patient was diagnosed with ET. a Bone marrow $(\mathrm{BM})$ aspirate showing increased megakaryocytes $(\times 200)$ and $\mathbf{b}$ frequent platelets $(\times 1000)$. c BM biopsy showing a normocellular marrow for age with increased megakaryocytes occurring in small clusters and include large forms with "cloud-like" atypical nuclei $(\times 400)$. d-f Representative images of a 9-year-old female with thrombocytosis and splenomegaly (WBC $9.9 \times 10^{9}$ cells $/ \mathrm{L}, \mathrm{Hgb}$ $13.7 \mathrm{~g} / \mathrm{dL}, \mathrm{MCV} 80.4 \mathrm{fL}$, platelet count $847 \times 10^{9}$ cells/L).

Adult patients with ET are at significant risk for the development of fibrosis, thrombosis, and leukemic progression. The risk of leukemia increases over time and is higher in the
Karyotype was normal. Molecular analysis was negative for $B C R$ $A B L$ but showed a $J A K 2 V 617 F$ mutation. The patient was initially diagnosed with MPN most consistent with ET based on the histologic findings but over time developed increasing Hgb with concern for possible polycythemia vera. d BM aspirate with clusters of atypical megakaryocytes $(\times 400)$. e BM biopsy shows a normocellular marrow for age with maturing myeloid and erythroid elements and increased eosinophils. Megakaryocytes are markedly increased and occurring in loose clusters. Large hyperlobated forms as well as condensed nuclear lobes are noted. Increased blasts (confirmed by CD34 and CD117 staining, not shown) were not noted $(\times 400)$. e Reticulin stain shows only minimal to mild diffused increase in reticulin $(\times 400)$

second and third decades after diagnosis [55, 56]. A recent pediatric study by Giona et al. addressed the risk for longterm complication in pediatric ET with a median follow-up
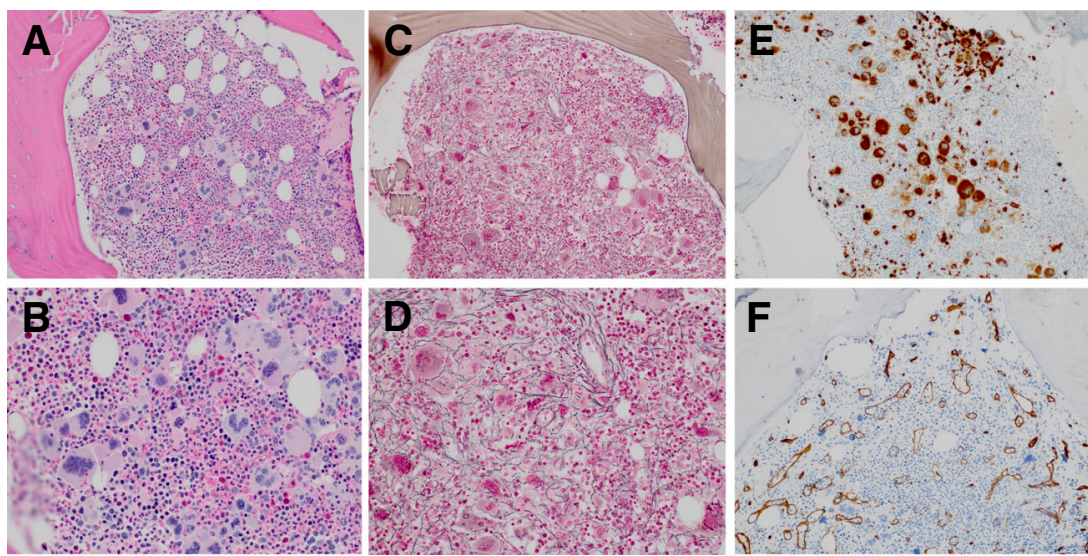

Fig. 2 Myeloproliferative neoplasm (MPN) due to CALR mutation. Images of a 14-year-old female presenting with marked thrombocytosis and mild leukocytosis with increased myeloid precursors and nucleated red blood cells on her peripheral smear. Blood counts at presentation showed WBC $11 \times 10^{9}$ cells/L (72\% neutrophils, $25 \%$ lymphocytes, $2 \%$ metamyelocytes, $1 \%$ atypical lymphocytes), Hgb $11.3 \mathrm{~g} / \mathrm{dL}, \mathrm{MCV} 73$, platelets $1218 \times 10^{9}$ cells/L. No splenomegaly present on exam. Karyotype was normal. Molecular analysis was negative for $B C R-A B L, J A K 2$ $V 617 F$, and $M P L$, but revealed a deletion type $C A L R$ mutation. The images show both features of essential thrombocythemia (ET) (large atypical often hyperlobated megakaryocytes) and primary myelofibrosis (PMF) (moderate increase in reticulin in increased vasculature) highlighting the fact that the histologic differential in these disorders is not always straightforward. a and $\mathbf{b}$ Display representative images of the bone marrow (BM) biopsy showing a markedly hypercellular marrow for age with an increased myeloid-to-erythroid ratio and a marked increase in megakaryocytes, which occur in larger clusters with marked variation in size. Small forms with condensed chromatin as well as large forms with abnormal chromatin clumping and occasional markedly hyperlobated forms are seen $(\times 200$ and $\times 400)$. c and d Reticulin stain shows overall diffused moderate increase in reticulin with marked perivascular fibrosis $(\times 200$ and $\times 400)$. e CD61 highlights markedly increased atypical megakaryocytes occurring in clusters. CD61 is also positive in associated platelets that are increased $(\times 200)$. f CD34 shows increased dilated vessels $(\times 200)$ 
Table 3 Diagnostic criteria for essential thrombocythemia (ET)

Diagnostic criteria (all 4 criteria must be met to establish a diagnosis of ET)

1. Sustained platelet count $\geq 450 \times 10^{9} / \mathrm{L}$

2. BM biopsy shows proliferation mainly of the megakaryocytic lineage with increased numbers of enlarged, mature megakaryocytes; no significant increase or left-shift of neutrophil granulopoiesis or erythropoiesis

3. Not meeting WHO criteria for PV, PMF, CML, MDS, or other myeloid neoplasm

4. Presence of $J A K 2 V 617 F$ or other clonal marker, or in the absence of clonal marker, no evidence for reactive thrombocytosis

Adapted from [72]

$B M$ bone marrow, $P V$ polycythemia vera, $P M F$ primary myelofibrosis, $C M L$ chronic myelogenous leukemia, $M D S$ myelodysplastic syndrome

time of over 10 years [25]. In this study, only a small fraction $(8 \%)$ of children developed thrombosis, all of whom had concurrent infections. Splenomegaly with progressive reticulin fibrosis was seen in $9 \%$ of patients at a median time of 108 months. The most feared complication - development of acute leukemia - was not observed in any patient. The risk of first-trimester miscarriages was low and similar to the general population.

\section{Primary myelofibrosis}

\section{Definitions and epidemiology}

Myelofibrosis (MF) - previously known as idiopathic MF, myeloid metaplasia with MF, or agnogenic myeloid metaplasia - can occur de novo (primary MF or PMF) or secondary to the development of marrow fibrosis after PV or ET (secondary MF) or in association with CML $[48,76]$.

PMF is characterized by PB cytopenias, leukoerythroblastosis, ineffective hematopoiesis, proliferation of dysfunctional megakaryocytes with reticulin and/or collagen fibrosis in the BM, and dysregulated cytokine production as well as intramedullary hematopoiesis (EMH) and hepatosplenomegaly. It carries the worst prognosis of the classical MPNs.

Adult PMF is the least common of the $\mathrm{Ph}-\mathrm{MPNs}$ with an incidence of $0.2-1$ per 100,000 persons per year $[52,61]$ and a male predominance. PMF is a disease of the older adult patient with a median age at diagnosis of 65-79 years and only $10 \%$ of cases diagnosed under the age of 45 years $[52,61]$.

PMF in children is exceedingly rare; only 42 total cases have been reported in the literature $[3,9,17,21,42,63,64$, $67,68]$. The largest study of pediatric PMF recently reported 19 patients from a single institution [17]. Given the rarity of the disease, precise epidemiologic data are lacking and the pediatric incidence is unknown. Based on the recent case series by DeLario et al., the median age at diagnosis of pediatric PMF is 14 months (range $0-17$ years) with a male predominance (1.7:1) [17]. It appears that pediatric PMF has a heterogeneous phenotype with variable outcomes ranging from spontaneous resolution to a rapidly progressive, sometimes fatal, disease curable only by HSCT. The clinical, histological, and molecular features of pediatric PMF differ from adult PMF. Importantly, a significant subset of pediatric patients came from consanguineous families with familial disease with more than one affected child in the pedigree [17, 67, 68]. This, along with the early onset of disease often in infancy, suggests that many cases represent hereditary myelofibrosis attributable to an autosomal recessive Mendelian trait.

\section{Pathophysiology}

Marrow fibrosis and EMH are the hallmarks of PMF. Clonal studies have confirmed the stem cell origin of the disease [33, 73]. The proliferation of clonal HSC leads to the production of excessive cytokines and growth factors within the BM niche. This process results in reactive proliferation of fibroblasts and mesenchymal cells $[6,29]$. The number of circulating HSCs $\left(\mathrm{CD} 34^{\text {low }}\right.$ cells) is significantly increased in PMF compared to other MPNs and even to other marrow stress with fibrosis, such as in secondary fibrosis due to metastatic carcinoma [5]. The number of circulating HSCs appears to be directly correlated with advanced BM fibrosis [7]. The clonal proliferation of megakaryocytes leads to overproduction of a number of growth factors, including: platelet-derived growth factor $(P D G F)$, transforming growth factor-B $(T G F b)$, epidermal growth factor $(E G F)$, and basic fibroblastic growth factor $(b F G F)$. This leads to the stromal reaction, which ultimately produces fibrosis. Furthermore, angiogenesis seems to play an important role in the disease process as an increased marrow vasculature is frequently observed in PMF $[29,74]$.

\section{Molecular genetics}

Somatic mutations in JAK2 V617F are found in $\sim 50 \%$ of adult patients. An additional $5-10 \%$ of patients have a somatic mutation in MPL (MPL W515K/L), leaving about $\sim 40 \%$ of PMF patients without an identified mutation (JAK2/MPLcases). Recently, somatic mutations in the $C A L R$ gene were identified in $35 \%$ of all PMF cases and in up to $88 \%$ of JAK2/ $M P L-$ PMF cases [36, 53]. All mutations identified were insertions or deletions in exon 9 of CALR causing frameshift mutations.

A recent NGS analysis of adult MPN patients revealed additional secondary mutations in ASXL1, EZH2, TET2, DNMT3A, and TP53 in PMF patients [40]. However, these secondary mutations also occurred in PV and ET and can be seen in MDS and other myeloid malignancies and are therefore not specific to PMF. Of the 34 PMF patients analyzed, 
$53 \%$ had one somatic mutation, $18 \%$ had two, and $15 \%$ had three concurrent somatic mutations [40]. An increased number of somatic mutations were associated with reduced overall survival and increased risk of leukemic transformation. TP53 mutations were associated with a particularly poor prognosis [36]. Other recent studies have shown that mutations in $A S X L 1$ and NRAS are of poor prognostic value [78, 85]. Whether these changes are present in pediatric PMF patients is unknown.

The underlying genetics of PMF in children remains largely undetermined. In the recent case series by DeLario et al., 17 BM samples of pediatric samples were tested for JAK2 V617F and $M P L W 515 K / L$, neither of which was detected in any sample [17]. The investigators also performed arraycomparative genomic hybridization (array-CGH) and identified various abnormalities in six children with PMF [17].

In a recent pediatric study of 14 children with PMF, CALR mutations were detected in $50 \%$ of the cases [4]. All of these mutations were type 2 variant $\left(K 385 f s^{*} 47\right)$, while type 1 variants $\left(2367 f f^{*} 46\right)$ were not detected. While there was no statistically significant difference in age, gender, and laboratory parameters between the CALR-mutated and CALR wild-type group, there appeared to be an association of CALR mutations in slightly older children (age 8-18 years). CALR mutations were not detected in the few young patients (age 2 years) tested [4].

The underlying genetic mechanism of pediatric PMF particularly in young infants with congenital myelofibrosis remains poorly understood. In 2013, two independent studies identified homozygous germline mutations in VPS45 in several families characterized by neutropenia, neutrophil dysfunction, BM fibrosis, progressive bone marrow failure, hepatosplenomegaly, and nephromegaly secondary to EMH $[70,88]$. No subsequent cases have been reported since then, supporting the fact that these are extremely rare conditions.

Cytogenetic studies in adult PMF show aberrations in 30$50 \%$ of the patients at diagnosis with an increase to $90 \%$ at the time of disease progression to AML. The most common cytogenetic changes include trisomies in chromosomes 8 and 9, del(13q), del(20q), del(12p), or trisomy 1q [31]. In contrast, cytogenetic changes in children with PMF are rarely detected.

\section{Clinical features}

Similar to adult PMF patients, the majority of children with PMF are symptomatic at diagnosis. However, in contrast to adult patients who frequently present with constitutional symptoms related to a cytokine-mediated hypercatabolic state, such as fatigue, weight loss, night sweats, fever, and pruritus, these symptoms are less frequently documented in children, although fever and fatigue have been observed [17]. The clinical presentation in children is often related to their cytopenias. In a recent case series containing 19 pediatric PMF patients, the majority of the patients had anemia $(95 \%, n=18)$ and thrombocytopenia ( $89 \%, n=17)$. Neutropenia $(37 \%, n=7)$ and eosinophilia was observed in $37 \%(n=7)$ and $10 \%(n=$ 10 ) of the cases, respectively. Leukoerythroblastosis, a common finding in adult patients with PMF and a minor criterion in the WHO diagnostic criteria (Table 4), was only observed in one child [17]. Organomegaly, a result of EMH, is frequently observed in both adults and children [17]. Other locations of EMH described in adult patients such as lymphadenopathy, vertebral column, lung, and skin involvement appear infrequently in children. Furthermore, EMH of the lungs can lead to pulmonary hypertension and is associated with a poor prognosis [66].

In general, children with PMF show a wide spectrum in their clinical presentation, some with a chronic indolent course and signs of cytopenias, others with a rapidly progressive and often fatal disorder more commonly observed in early infancy $[67,68]$.

\section{Differential diagnosis}

The WHO diagnostic criteria for PMF are outlined in Table 4 and have largely been developed with the clinical, laboratory, and molecular features found in adult PMF. As discussed, $J A K 2$ V617F and MPL mutations are extremely rare and in some reports absent in children with PMF. Therefore, fulfilling the third major PMF criterion is unlikely. Other clonal markers in children rarely exist. A few cases with varying array-CGH abnormalities have been described but their clinical significance is uncertain [17]. Given that the molecular underpinnings in pediatric PMF are not known, the diagnosis in children must rely heavily on excluding alternate causes (Table 5). Careful clinical and laboratory assessment and evaluation of the bone marrow pathology are essential to make an accurate diagnosis and initiating the appropriate therapy. Interval follow-up is often required to confirm the diagnosis or rule out more acute disorders, such as acute megakaryoblastic leukemia, AML-M7 (acute myelofibrosis), MDS with myelofibrosis or acute lymphoblastic leukemia (ALL). Ancillary laboratory and molecular and histopathology studies can aid in the diagnostic work up for children (Table 6).

Given that the fibrotic marrow frequently cannot be aspirated, immunohistochemistry (IHC) studies and special stains may be required. CD34 and CD117 in conjunction with lineage-specific markers aid in the characterization of increased blast populations and help differentiate PMF from AML-M7 and ALL. CD34 also highlights the increased vasculature and dilated sinusoids often seen in PMF. IHC for CD3, CD20, CD4, and CD8 can highlight lymphoid aggregates and is important in ruling out autoimmune myelofibrosis (AIMF), a disorder characterized by marrow fibrosis, lymphoid aggregates, and a benign clinical course. Patients with 
Table 4 Diagnostic criteria for primary myelofibrosis

Major criteria

All criteria must be met
Minor criteria

At least 2 criteria must be met

1. Leukoerythroblastosis

2. Increased serum lactate dehydrogenase (LDH)

3. Anemia

4. Palpable splenomegaly

Adapted from [72]

$B M$ bone marrow, $P V$ polycythemia vera, $C M L$ chronic myelogenous leukemia, $M D S$ myelodysplastic syndrome

AIMF typically show mild fibrosis, a hypercellular marrow with erythroid and megakaryocytic hyperplasia, and no dysplasia or megakaryocytic atypia. Lymphoid aggregates are usually non-paratrabecular and $\mathrm{T}$ cell prominent but can show a mixed $\mathrm{B}$ and $\mathrm{T}$ cell phenotype. Mild polytypic plasmocytosis can be observed as well [87].

\section{Laboratory and histopathology features}

Anemia is the most common laboratory abnormality at presentation, followed by thrombocytopenia. Neutropenia is less

Table 5 Differential diagnosis of primary myelofibrosis in children

Malignant/Clonal myeloid disorders
Acute megakaryoblastic leukemia, AML-M7 (Acute myelofibrosis)
Myelodysplastic syndromes with myelofibrosis ${ }^{\mathrm{a}}$
Noonan-like syndrome due to CBL mutation
Late-stage PV, ET, or CML with evolution to fibrosis
Malignant causes (non-myeloid) of secondary myelofibrosis
Hodgkin lymphoma
Non-Hodgkin lymphoma
Acute lymphoblastic leukemia
Systemic mastocytosis
Hemaphagocytic lymphohistiocytosis
Non-malignant disorders of myelofibrosis
Primary autoimmune myelofibrosis
Autoimmune disorders (e.g., systemic lupus, arthritis, autoimmune
hemolytic anemia) with secondary myelofibrosis
Infections (granulomatous infections such as tuberculosis and
histoplasmosis)
Renal osteopathy
Osteopetrosis
Other non-malignant conditions
Vitamin D deficiency

${ }^{\mathrm{a}}$ Including patients with GATA2 mutation, in particular those with monosomy 7 common and observed in approximately one third of patients [17]. Studies on precise laboratory values at presentation of pediatric PMF are not available, but the available case reports suggest $\mathrm{Hgb}$ and platelet values in the range of 4.9-9.4 $\mathrm{g} / \mathrm{dL}$ and $23-242 \times 10^{9} / \mathrm{L}$, respectively [30]. Other laboratory values commonly increased in adult PMF patients - including LDH, bilirubin, and uric acid levels suggesting high marrow cell turnover - are not well documented in children.

The PB smear requires careful evaluation for signs of marrow distress. While an overt leukoerythroblastic pictureclassical in adult PMF - is not always observed to the same degree in children [17], signs of marrow stress such as occasional nucleated red blood cells, scattered teardrop erythrocytes, and left shifted myeloid cells with increased myeloid precursors may be noted. Platelets can show variable sizes including large forms.

The histopathologic features present in adult PMF, including hyerpcellularity, megakaryocytic hyperplasia, granulocyte proliferation, and decreased erythroid precursors, are also present in pediatric PMF $[1,17,83,84]$. A systematic review of 19 pediatric PMF cases demonstrated hypercellular marrows with megakaryocytic hyperplasia and moderate to severe reticulin fibrosis. The myeloid-to-erythroid ratio was typically increased $(n=10)$. Eosinophilia was observed in $40 \%$ of the patients. Megakaryocytic dysplasia was observed in $40 \%$ and characterized by hypolobated forms, separation of nuclear lobes, and micromegakaryocytes [17]. The classical megakaryocytic dysplasia described in adult PMF, such as hyperchromatic, smudgy, bulbous, and cloud-like nuclei, were not observed in this series but have been commented upon in other pediatric PMF cases as demonstrated in Fig. 3. IHC staining for CD61 can be helpful to highlight atypical and identify micromegakaryocytes. IHC for CD34 and CD117 (c-kit) should be considered to determine if blasts are increased, indicative of leukemic progression. The majority of pediatric PMF patients show moderate to severe reticulin fibrosis (grades 2-3), observed in $74 \%$ of the patients, while the 
Table 6 Suggested diagnostic work up for pediatric patients with suspected primary myelofibrosis

\begin{tabular}{ll}
\hline Diagnostic checklist & \\
\hline Basic laboratory & $\checkmark$ CBC with differential \\
& $\checkmark$ Reticulocyte count \\
& $\checkmark$ Basic chemistry studies (electrolytes, LDH, uric acid) \\
& $\checkmark$ Renal and liver function tests \\
& $\checkmark$ Hemoglobin F (hemoglobin electrophoresis) \\
& $\checkmark$ Lymphocyte subsets (B and T cell subsets) \\
& $\checkmark$ Autoimmune work up (ANA, DAT, RF etc.) \\
& $\checkmark$ High-resolution HLA typing \\
& $\checkmark$ Nutritional deficiencies (vitamin D) \\
& $\checkmark$ Infectious studies (HIV, tuberculosis, histoplasmosis, \\
& leishmaniasis, etc.) \\
& $\checkmark$ Noonan-like syndrome due to CBL mutation \\
Other laboratory & $\checkmark$ VPS45 \\
& $\checkmark$ JAK2, MPL, CALR \\
& $\checkmark$ GATA2 \\
& $\checkmark$ Peripheral blood smear review \\
Inherited & $\checkmark$ Bone marrow aspirate for morphology \\
Conditions/Syndromes & $\checkmark$ Bone marrow biopsy for histology (consider bilateral) \\
Molecular diagnostics & $\checkmark$ IHC staining (consider CD34, CD117, CD61, TdT, CD3, \\
& CD20, CD4, CD8, CD138) \\
Histopathology & $\checkmark$ Reticulin stain \\
& $\checkmark$ Flow cytometry (in case of excess blasts) \\
& $\checkmark$ Karyotype form BM \\
& $\checkmark$ FISH on BM (-7, 7q-, +8, -5, 5q-, 20q-) \\
Additional studies & \\
\hline &
\end{tabular}

$A N A$ antinuclear antibody, $D A T$ direct antiglobulin titers, $R F$ rheumatoid factor remaining patients show mild (grade 1) fibrosis [17]. Fibrosis can be patchy and associated with the clustering of megakaryocytes (Fig. 3) or diffused. Grades 1-2 collagen fibrosis highlighted by trichrome staining has been observed in $37 \%$ of pediatric PMF [17]. Significant osteosclerosis has not been observed [17].

A diagnostic BM aspirate is often difficult to obtain given the marked marrow fibrosis. This can make the differential diagnosis of MDS with fibrosis challenging, as a highquality aspirate is required to assess cytologic dysplasia.

\section{Treatment, clinical outcome, and prognosis}

In adult PMF patients, treatment approaches are largely based on palliative and supportive care. A number of cytoreductive agents have been used in adults, including hydroxyurea, busulfan, 6-mercaptopurine, and 2-chlorodeoxyadenosine (2$\mathrm{CDA}$ /cladribine), interferon- $\alpha$, lenalidomide, and thalidomide (in adults with 5q- and PMF). Other agents such as corticosteroids, androgens, danazol, as well as hematopoietic growth factors have been used as well. Recently, JAK2 inhibitors are becoming the first line of therapy in most patients and producing markedly improved outcome. None of these agents have been studied in children and are not routinely used.
Supportive care with red cell and platelet transfusions should be considered in children with PMF and is guided by the degree of cytopenia and symptomatology until definitive therapy with HSCT can be performed.

Progression to AML, which is the cause of death in 5-30\% of adult PMF patients, is rarely observed in children with PMF [17].

HSCT is the only curative therapy for PMF and has been reserved for younger adult individuals with poor prognostic features. In pediatric patients with a confirmed diagnosis, most investigators have rapidly referred to HSCT to avoid delaying definitive therapy and secondary complications such as infections and transfusion dependency [17, 21].

Outcomes after HSCT are reported in single or small case series reports with heterogeneous donors, stem cell sources, and conditioning regimens [10, 17, 21, 30, 51, 62, 64-66, 71]. Almost all reported cases are those of young children ( $<3$ years) and infants. Overall, the HSCT outcomes for these patients were favorable with 21 of 28 transplanted patients surviving disease-free. Six patients were deceased and one patient had insufficient follow-up with pending engraftment at the time of the case report. Three out of the 28 children were infants with VPS45 mutation and myelofibrosis of which two survived after HSCT [71]. 

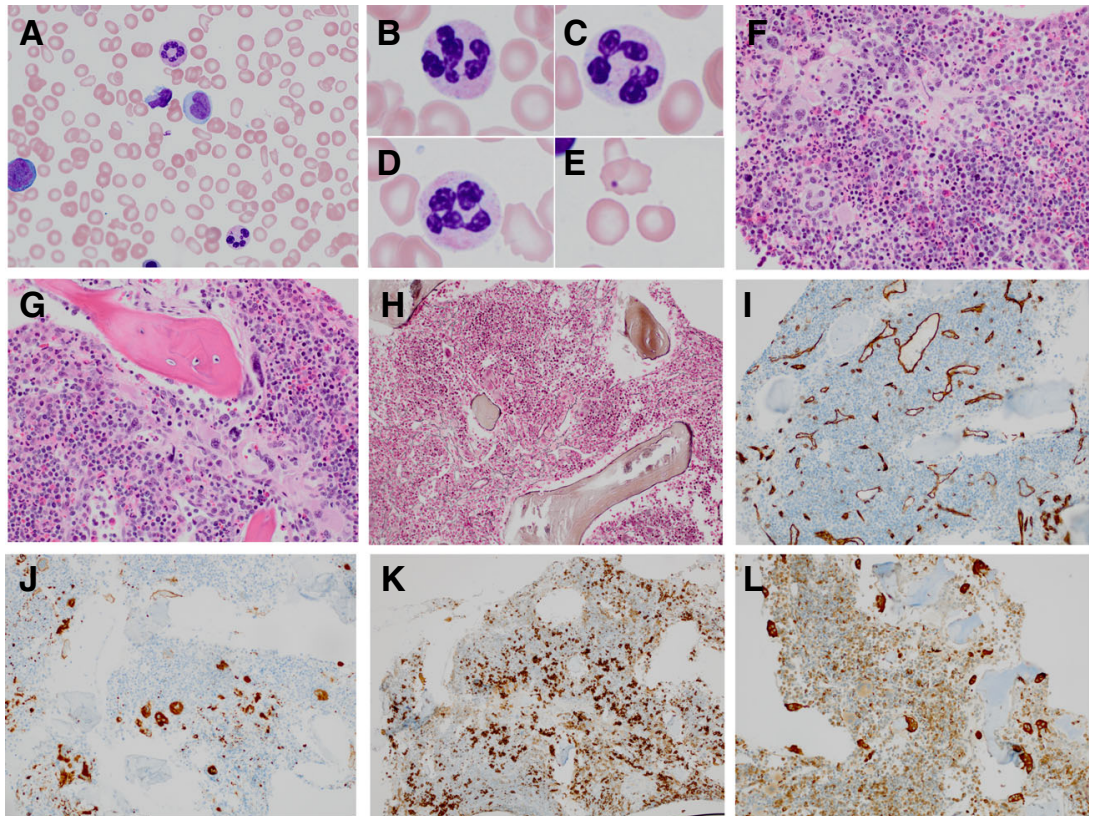

Fig. 3 Congenital familial myelofibrosis. Representative images from the bone marrow $(\mathrm{BM})$ aspirate and biopsy of a 10-month-old male with the diagnosis of primary congenital familial myelofibrosis. The patient presented with easy bruising and petechiae on the second day of life. $\mathrm{He}$ had an older brother with congenital myelofibrosis leading to high suspicion for a familial disorder. Blood counts showed mild leukocytosis of $12.56 \times 10^{9}$ cells $/ \mathrm{L}$ with $43 \%$ neutrophils, $41 \%$ lymphocytes, $12 \%$ monocytes, $2 \%$ eosinophils, and $3 \%$ basophils. The Hgb was $8.4 \mathrm{~g} / \mathrm{dL}$ and the platelet count was $17 \times 10^{9}$ cells/L. Cytogenetic and FISH studies showed a normal karyotype and were negative for monosomy 7 , trisomy 8, and 5q-. Molecular studies were negative for JAK2 V617F, MPL, and VPS45 mutations. a-e The BM was aspicular and hypocellular secondary to difficult aspiration. Maturing myeloid and erythroid elements were noted with many hyperlobated neutrophils. Vitamin B12 and folate deficiency were ruled out. Background mature erythrocytes show anisiopoikilocytosis including microcytes, macrocytes, target cells,

It is important to note that successful HSCT leads to the complete resolution of myelofibrosis with normal hematopoiesis. However, the histologic reversal of marrow fibrosis took time with improvements noted around 3 months and resolution typically by 1 year.

Occasional cases of spontaneous remissions, long-term stable disease, and resolution after a course of steroids have been described [3, 15, 17, 39, 41, 54, 58, 63, 64, 69], suggesting that some of the cases diagnosed as PMF might in fact have been primary or secondary AIMF. While there are some recent case studies comparing PMF to AIMF and proving guidance differentiating the two conditions, this differential remains challenging in children [87]. If a trial of steroid therapy is considered in a pediatric patient with PMF, it is important to rule out a smoldering leukemia, namely, ALL and AML-M7, with the highest confidence in order to avoid masking an acute leukemia further. Furthermore, a trial of vitamin D should be considered in pediatric patients with myelofibrosis and low vitamin $\mathrm{D}$ levels $[2,28,91]$ prior to considering more aggressive management of these patients. elliptocytes, pencil cells, acanthocytes, and teardrop forms. Hypochromasia and Howell-Jolly body-like inclusions are also noted (×1000). $\mathbf{f}-\mathbf{l}$ Representative BM biopsy from the same patient. $\mathbf{f}$ and $\mathbf{g}$ shows H\&E images demonstrating a normo- to hypercellular marrow for age with moderate megakaryocytic hyperplasia occurring in loose clusters with dysmorphic features, including large forms, cloud-like nuclei, and occasional small forms $(\times 400)$. $\mathbf{h}$ Reticulin was moderately increased in particular in association with the megakaryocyte clusters $(\times 200)$. i Immunohistochemistry $(\mathrm{ICH})$ for CD34 shows no increase in blasts but vascular proliferation, focal liminal distension, and occasional intrasinusoidal hematopoietic elements $(\times 200)$. j CD61 highlights clusters of atypical megakaryocytes with associated increase in platelets $(\times 200)$. k CD71 highlights minimally decreased erythroid precursors $(\times 200)$. I CD68 is positive in scattered histiocytes but also highlights osteoclasts $(\times 200)$

No molecular testing is available to confirm the diagnosis of pediatric PMF. Furthermore, it is difficult to predict the disease evolution. Some children appear to show spontaneous regression or a long-term stable disease course. Other patients, in particular, young children with evidence of familial or congenital myelofibrosis, have been shown to have rapidly progressive disease leading to death early in life $[67,68]$. For those patients expedited HSCT is indicated.

The only gene recently identified in pediatric PMF cases is VPS45. In many ways the disorder appears to be a distinct entity with immunodeficiency and other congenital abnormalities [70, 88].

Prognostic scoring systems such as the Dynamic International Prognostic Scoring System (DIPSS) frequently used for risk stratification in adult PMF have not been employed for pediatric PMF patients. This is largely because some of the important risk factors such as age $(>65)$ and unfavorable cytogenetics are not present in children. Furthermore, pediatric PMF is rare and therefore precludes a 
systematic analysis of any prognostic scoring system in this population.

\section{Conclusions}

Classical $\mathrm{Ph}-\mathrm{MPNs}$ are exceedingly rare disorders in children. While PV and ET appear to occur in older children, most pediatric PMF cases occur in infants and young children.

The majority of adult MPNs carry mutation in $J A K 2, M P L$, and CALR. However, the majority of children with MPN do not carry any of those mutations and the underlying genetics remain largely unknown. Given the high proportion of familial and congenital cases, in particular in PMF, we can assume that many children have an inherited predisposition caused by germline mutations likely inherited in an autosomal recessive manner. The recent discovery of VPS45 mutations causing a unique syndrome of myelofibrosis, marrow failure, and immunodeficiency supports this hypothesis.

The majority of children and adolescents with PV and ET are managed with supportive care and sometimes cytoreductive therapies. Although occasional spontaneous regression of PMF has been observed, most children with PMF require HSCT and have good overall outcomes.

Given the rarity of MPNs in children, national and international collaborative efforts to study MPN in pediatric patients would aid in our understanding of these disorders and could facilitate new insight into genetic discovery through the use of NGS technologies.

Ethical approval This article does not contain any studies with human participants or animals performed by any of the authors.

Conflict of interest Author IH declares that she has no conflict of interest.

Funding This study was funded by the National Institutes of Health career development award from the National Cancer Institute (1K08CA140723).

\section{References}

1. Ahmed A, Chang CC (2006) Chronic idiopathic myelofibrosis: clinicopathologic features, pathogenesis, and prognosis. Arch Pathol Lab Med 130:1133-1143. doi:10.1043/1543-2165(2006) 130[1133:CIM]2.0.CO;2

2. Akyay A, Solmaz OA (2012) Myelofibrosis associated with vitamin D deficiency rickets in an older aged child. Pediatr Hematol Oncol 29:691-693. doi:10.3109/08880018.2012.727064

3. Altura RA, Head DR, Wang WC (2000) Long-term survival of infants with idiopathic myelofibrosis. Br J Haematol 109:459-462

4. An W, Wan Y, Guo Y, Chen X, Ren Y, Zhang J, Chang L, Wei W, Zhang P, Zhu X (2014) CALR mutation screening in pediatric primary myelofibrosis. Pediatr Blood Cancer 61:2256-2262. doi: $10.1002 /$ pbc. 25211
5. Andreasson B, Swolin B, Kutti J (2002) Patients with idiopathic myelofibrosis show increased CD34+ cell concentrations in peripheral blood compared to patients with polycythaemia vera and essential thrombocythaemia. Eur J Haematol 68:189-193

6. Barosi G (1999) Myelofibrosis with myeloid metaplasia: diagnostic definition and prognostic classification for clinical studies and treatment guidelines. J Clin Oncol 17:2954-2970

7. Barosi G, Viarengo G, Pecci A, Rosti V, Piaggio G, Marchetti M, Frassoni F (2001) Diagnostic and clinical relevance of the number of circulating CD34(+) cells in myelofibrosis with myeloid metaplasia. Blood 98:3249-3255

8. Berlin NI (2002) Polycythemia vera: diagnosis and treatment 2002. Expert Rev Anticancer Ther 2:330-336. doi:10.1586/14737140.2. 3.330

9. Boxer LA, Camitta BM, Berenberg W, Fanning JP (1975) Myelofibrosis-myeloid metaplasia in childhood. Pediatrics 55: 861-865

10. Cahn JY, Plouvier E, Flesch M, Carbillet JP, Herve P (1987) T celldepleted allogeneic bone marrow transplantation in a case of childhood idiopathic myelofibrosis. Bone Marrow Transplant 2:209-211

11. Carbuccia N, Murati A, Trouplin V, Brecqueville M, Adelaide J, Rey J, Vainchenker W, Bernard OA, Chaffanet M, Vey N et al (2009) Mutations of ASXL1 gene in myeloproliferative neoplasms. Leukemia 23:2183-2186. doi:10.1038/leu.2009.141

12. Cario H (2005) Childhood polycythemias/erythrocytoses: classification, diagnosis, clinical presentation, and treatment. Ann Hematol 84:137-145. doi:10.1007/s00277-004-0985-1

13. Cario H, Schwarz K, Herter JM, Komrska V, McMullin MF, Minkov M, Niemeyer C, Pospisilova D, Reinhard H, Debatin $\mathrm{KM}$ et al (2008) Clinical and molecular characterisation of a prospectively collected cohort of children and adolescents with polycythemia vera. Br J Haematol 142:622-626. doi:10.1111/j.13652141.2008.07220.x

14. Cervantes F (2011) Management of essential thrombocythemia. Hematology Am Soc Hematol Educ Program 2011:215-221. doi: 10.1182/asheducation-2011.1.215

15. Cetingul N, Yener E, Oztop S, Nisli G, Soydan S (1994) Agnogenic myeloid metaplasia in childhood: a report of two cases and efficiency of intravenous high dose methylprednisolone treatment. Acta Paediatr Jpn 36:697-700

16. Chim CS, Kwong YL, Lie AK, Ma SK, Chan CC, Wong LG, Kho BC, Lee HK, Sim JP, Chan CH et al (2005) Long-term outcome of 231 patients with essential thrombocythemia: prognostic factors for thrombosis, bleeding, myelofibrosis, and leukemia. Arch Intern Med 165:2651-2658. doi:10.1001/archinte.165.22.2651

17. DeLario MR, Sheehan AM, Ataya R, Bertuch AA, Vega C 2nd, Webb CR, Lopez-Terrada D, Venkateswaran L (2012) Clinical, histopathologic, and genetic features of pediatric primary myelofibrosis-an entity different from adults. Am J Hematol 87:461-464. doi:10.1002/ajh.23140

18. Delhommeau F, Dupont S, Della Valle V, James C, Trannoy S, Masse A, Kosmider O, Le Couedic JP, Robert F, Alberdi A et al (2009) Mutation in TET2 in myeloid cancers. N Engl J Med 360: 2289-2301. doi:10.1056/NEJMoa0810069

19. Ding J, Komatsu H, Wakita A, Kato-Uranishi M, Ito M, Satoh A, Tsuboi K, Nitta M, Miyazaki H, Iida S et al (2004) Familial essential thrombocythemia associated with a dominant-positive activating mutation of the c-MPL gene, which encodes for the receptor for thrombopoietin. Blood 103:4198-4200. doi:10.1182/blood-200310-3471

20. Dobo I, Boiret N, Lippert E, Girodon F, Mossuz P, Donnard M, Campos L, Pineau D, Bascans E, Praloran V et al (2004) A standardized endogenous megakaryocytic erythroid colony assay for the diagnosis of essential thrombocythemia. Haematologica 89: $1207-1212$ 
21. Domm J, Calder C, Manes B, Crossno C, Correa H, Frangoul H (2009) Unrelated stem cell transplant for infantile idiopathic myelofibrosis. Pediatr Blood Cancer 52:893-895. doi:10.1002/pbc. 21910

22. Dror Y, Zipursky A, Blanchette VS (1999) Essential thrombocythemia in children. J Pediatr Hematol Oncol 21:356-363

23. Fu R, Zhang L, Yang R (2013) Paediatric essential thrombocythaemia: clinical and molecular features, diagnosis and treatment. Br J Haematol 163:295-302. doi:10.1111/bjh.12530

24. Gangat N, Wolanskyj AP, McClure RF, Li CY, Schwager S, Wu W, Tefferi A (2007) Risk stratification for survival and leukemic transformation in essential thrombocythemia: a single institutional study of 605 patients. Leukemia 21:270-276. doi:10.1038/sj.leu.2404500

25. Giona F, Teofili L, Moleti ML, Martini M, Palumbo G, Amendola A, Mazzucconi MG, Testi AM, Pignoloni P, Orlando SM et al (2012) Thrombocythemia and polycythemia in patients younger than 20 years at diagnosis: clinical and biologic features, treatment, and long-term outcome. Blood 119:2219-2227. doi:10.1182/blood2011-08-371328

26. Harrison CN, Campbell PJ, Buck G, Wheatley K, East CL, Bareford D, Wilkins BS, van der Walt JD, Reilly JT, Grigg AP et al (2005) Hydroxyurea compared with anagrelide in high-risk essential thrombocythemia. N Engl J Med 353:33-45. doi:10.1056/ NEJMoa043800

27. Hasle H (2000) Incidence of essential thrombocythaemia in children. Br J Haematol 110:751

28. Henry M, Chitlur M, Rajpurkar M, Mastropietro CW, Poulik J, Ravindranath Y (2014) Myelofibrosis, hepatic extramedullary hematopoiesis and ascites associated with vitamin D deficiency in early infancy. J Pediatr Hematol Oncol 36:319-321. doi:10.1097/ MPH.0b013e31828e548a

29. Hoffman R, Xu M, Finazzi G, Barbui T (2008) Primary myelofibrosis. In: Hoffman R, Benz EJ, Shattil SJ (eds) Hematology: basic principles and practice, 5th edn. Churchill Livingstone, Philadelphia, Pa, pp 1125-1148

30. Hussein AA, Hamadah T, Domm J, Al-Zaben A, Frangoul H (2013) Allogeneic hematopoietic stem cell transplantation for infants with idiopathic myelofibrosis. Pediatr Transplant 17:815-819. doi:10.1111/petr.12148

31. Hussein K, Van Dyke DL, Tefferi A (2009) Conventional cytogenetics in myelofibrosis: literature review and discussion. Eur $\mathrm{J}$ Haematol 82:329-338. doi:10.1111/j.1600-0609.2009.01224.x

32. Ismael O, Shimada A, Hama A, Sakaguchi H, Doisaki S, Muramatsu H, Yoshida N, Ito M, Takahashi Y, Akita N et al (2012) Mutations profile of polycythemia vera and essential thrombocythemia among Japanese children. Pediatr Blood Cancer 59:530-535. doi:10.1002/pbc.23409

33. Jacobson RJ, Salo A, Fialkow PJ (1978) Agnogenic myeloid metaplasia: a clonal proliferation of hematopoietic stem cells with secondary myelofibrosis. Blood 51:189-194

34. Jensen MK, de Nully BP, Nielsen OJ, Hasselbalch HC (2000) Incidence, clinical features and outcome of essential thrombocythaemia in a well defined geographical area. Eur J Haematol 65:132-139

35. Kelly K, McMahon C, Langabeer S, Eliwan H, O'Marcaigh A, Smith OP (2008) Congenital JAK2V617F polycythemia vera: where does the genotype-phenotype diversity end? Blood 112: 4356-4357. doi:10.1182/blood-2008-08-175620

36. Klampfl T, Gisslinger H, Harutyunyan AS, Nivarthi H, Rumi E, Milosevic JD, Them NC, Berg T, Gisslinger B, Pietra D et al (2013) Somatic mutations of calreticulin in myeloproliferative neoplasms. N Engl J Med 369:2379-2390. doi:10.1056/NEJMoa1311347

37. Kucine N, Chastain KM, Mahler MB, Bussel JB (2014) Primary thrombocytosis in children. Haematologica 99:620-628. doi:10. 3324/haematol.2013.092684
38. Lackner H, Urban C, Benesch M, Moser A, Sovinz P, Schwinger W, Dornbusch HJ (2006) Long-term use of anagrelide in the treatment of children with essential thrombocythemia. Eur J Haematol 77:358-359. doi:10.1111/j.1600-0609.2006.00719.x

39. Lau SO, Ramsay NK, Smith CM 2nd, McKenna R, Kersey JH (1981) Spontaneous resolution of severe childhood myelofibrosis. J Pediatr 98:585-588

40. Lundberg P, Karow A, Nienhold R, Looser R, Hao-Shen H, Nissen I, Girsberger S, Lehmann T, Passweg J, Stern M et al (2014) Clonal evolution and clinical correlates of somatic mutations in myeloproliferative neoplasms. Blood 123:2220-2228. doi:10.1182/blood2013-11-537167

41. Macdougall LG, Pettifor JM, Patel JM (1987) Bone growth and haemopoiesis: steroid reversible anaemia, myelofibrosis and increased bone formation in a child. Br J Haematol 66:5-10

42. Mallouh AA, Sa'di AR (1992) Agnogenic myeloid metaplasia in children. Am J Dis Child 146:965-967

43. Marchioli R, Finazzi G, Landolfi R, Kutti J, Gisslinger H, Patrono C, Marilus R, Villegas A, Tognoni G, Barbui T (2005) Vascular and neoplastic risk in a large cohort of patients with polycythemia vera. J Clin Oncol 23:2224-2232. doi:10.1200/JCO.2005.07.062

44. Mazzucconi MG, Redi R, Bernasconi S, Bizzoni L, Dragoni F, Latagliata R, Santoro C, Mandelli F (2004) A long-term study of young patients with essential thrombocythemia treated with anagrelide. Haematologica 89:1306-1313

45. McNally RJ, Rowland D, Roman E, Cartwright RA (1997) Age and sex distributions of hematological malignancies in the U.K. Hematol Oncol 15:173-189

46. Mead AJ, Rugless MJ, Jacobsen SE, Schuh A (2012) Germline JAK2 mutation in a family with hereditary thrombocytosis. N Engl J Med 366:967-969. doi:10.1056/NEJMc1200349

47. Mesa RA, Silverstein MN, Jacobsen SJ, Wollan PC, Tefferi A (1999) Population-based incidence and survival figures in essential thrombocythemia and agnogenic myeloid metaplasia: an Olmsted County Study, 1976-1995. Am J Hematol 61:10-15

48. Mesa RA, Verstovsek S, Cervantes F, Barosi G, Reilly JT, Dupriez B, Levine R, Le Bousse-Kerdiles MC, Wadleigh M, Campbell PJ et al (2007) Primary myelofibrosis (PMF), post polycythemia vera myelofibrosis (post-PV MF), post essential thrombocythemia myelofibrosis (post-ET MF), blast phase PMF (PMF-BP): consensus on terminology by the international working group for myelofibrosis research and treatment (IWG-MRT). Leuk Res 31:737-740. doi:10. 1016/j.leukres.2006.12.002

49. Mi JQ, Blanc-Jouvan F, Wang J, Sotto MF, Cousin F, Castinel A, Chauvet M, Sotto JJ, Polack B, Mossuz P (2001) Endogenous megakaryocytic colony formation and thrombopoietin sensitivity of megakaryocytic progenitor cells are useful to distinguish between essential thrombocythemia and reactive thrombocytosis. J Hematother Stem Cell Res 10:405-409. doi:10.1089/ 152581601750289000

50. Michiels JJ, Juvonen E (1997) Proposal for revised diagnostic criteria of essential thrombocythemia and polycythemia vera by the Thrombocythemia Vera Study Group. Semin Thromb Hemost 23:339-347. doi:10.1055/s-2007-996107

51. Mitton B, de Oliveira S, Pullarkat ST, Moore TB (2013) Stem cell transplantation in primary myelofibrosis of childhood. J Pediatr Hematol Oncol 35:e120-e122. doi:10.1097/MPH. 0b013e31828800cc

52. Moulard O, Mehta J, Fryzek J, Olivares R, Iqbal U, Mesa RA (2014) Epidemiology of myelofibrosis, essential thrombocythemia, and polycythemia vera in the European Union. Eur J Haematol 92: 289-297. doi:10.1111/ejh.12256

53. Nangalia J, Massie CE, Baxter EJ, Nice FL, Gundem G, Wedge DC, Avezov E, Li J, Kollmann K, Kent DG et al (2013) Somatic CALR mutations in myeloproliferative neoplasms with nonmutated 
JAK2. N Eng1 J Med 369:2391-2405. doi:10.1056/ NEJMoa1312542

54. Ozsoylu S (1993) Megadose methylprednisolone for myelofibrosis. Acta Haematol 89:167

55. Passamonti F, Rumi E, Arcaini L, Boveri E, Elena C, Pietra D, Boggi S, Astori C, Bernasconi P, Varettoni M et al (2008) Prognostic factors for thrombosis, myelofibrosis, and leukemia in essential thrombocythemia: a study of 605 patients. Haematologica 93:1645-1651. doi:10.3324/haematol.13346

56. Passamonti F, Rumi E, Pungolino E, Malabarba L, Bertazzoni P, Valentini M, Orlandi E, Arcaini L, Brusamolino E, Pascutto C et al (2004) Life expectancy and prognostic factors for survival in patients with polycythemia vera and essential thrombocythemia. Am J Med 117:755-761. doi:10.1016/j.amjmed.2004.06.032

57. Pearson TC, Messinezy M (2001) Idiopathic erythrocytosis, diagnosis and clinical management. Pathol Biol 49:170-177

58. Pilorget H, Bangui A, Adam M, Leverger G (1996) Myelofibrosis regressing under corticotherapy and intravenous immunoglobulins in an infant. Arch Pediatr 3:40-43

59. Randi ML, Putti MC, Scapin M, Pacquola E, Tucci F, Micalizzi C, Zanesco L, Fabris F (2006) Pediatric patients with essential thrombocythemia are mostly polyclonal and V617FJAK2 negative. Blood 108:3600-3602. doi:10.1182/blood-2006-04-014746

60. Reinhard H, Klingebiel T, Lang P, Bader P, Niethammer D, Graf N (2008) Stem cell transplantation for polycythemia vera. Pediatr Blood Cancer 50:124-126. doi:10.1002/pbc.20906

61. Rollison DE, Howlader N, Smith MT, Strom SS, Merritt WD, Ries LA, Edwards BK, List AF (2008) Epidemiology of myelodysplastic syndromes and chronic myeloproliferative disorders in the United States, 2001-2004, using data from the NAACCR and SEER programs. Blood 112:45-52. doi:10.1182/blood-200801-134858

62. Rossbach HC, Grana NH, Chamizo W, Barrios NJ, Barbosa JL (1996) Successful allogeneic bone marrow transplantation for agnogenic myeloid metaplasia in a 3-year-old boy. J Pediatr Hematol Oncol 18:213-215

63. Sah A, Minford A, Parapia LA (2001) Spontaneous remission of juvenile idiopathic myelofibrosis. Br J Haematol 112:1083

64. Sekhar M, Prentice HG, Popat U, Anderson D, Janmohammed R, Roberts I, Britt RP (1996) Idiopathic myelofibrosis in children. Br J Haematol 93:394-397

65. Shaikh F, Naithani R, Kirby-Allen M, Doyle J (2012) Allogeneic cord hematopoietic stem cell transplantation in an infant with primary myelofibrosis. J Pediatr Hematol Oncol 34:e199-e201. doi: 10.1097/MPH.0b013e3182346cc5

66. Shankar S, Choi JK, Dermody TS, Head DR, Bunin N, Iannone R (2004) Pulmonary hypertension complicating bone marrow transplantation for idiopathic myelofibrosis. J Pediatr Hematol Oncol 26: 393-397

67. Sheikha A (2004) Fatal familial infantile myelofibrosis. J Pediatr Hematol Oncol 26:164-168

68. Sieff CA, Malleson P (1980) Familial myelofibrosis. Arch Dis Child 55:888-893

69. Slone JS, Smith MC, Seegmiller AC, Sidonio RF, Yang E (2013) Idiopathic myelofibrosis in children: primary myelofibrosis, essential thrombocythemia, or transient process? J Pediatr Hematol Oncol 35:559-565. doi:10.1097/MPH.0b013e31829cdea0

70. Stepensky P, Saada A, Cowan M, Tabib A, Fischer U, Berkun Y, Saleh H, Simanovsky N, Kogot-Levin A, Weintraub M et al (2013) The Thr224Asn mutation in the VPS45 gene is associated with the congenital neutropenia and primary myelofibrosis of infancy. Blood 121:5078-5087. doi:10.1182/blood-2012-12-475566

71. Stepensky P, Simanovsky N, Averbuch D, Gross M, Yanir A, Mevorach D, Elpeleg O, Weintraub M (2013) VPS 45-associated primary infantile myelofibrosis-successful treatment with hematopoietic stem cell transplantation. Pediatr Transplant 17: 820-825. doi:10.1111/petr.12169

72. Swerdlow SH, International Agency for Research on Cancer, World Health Organization (2008) WHO classification of tumours of haematopoietic and lymphoid tissues. International Agency for Research on Cancer, IARC, Lyon

73. Tefferi A (2000) Myelofibrosis with myeloid metaplasia. N Engl J Med 342:1255-1265. doi:10.1056/NEJM200004273421706

74. Tefferi A (2005) Pathogenesis of myelofibrosis with myeloid metaplasia. J Clin Oncol 23:8520-8530. doi:10.1200/JCO.2004.00.9316

75. Tefferi A, Guglielmelli P, Lasho TL, Rotunno G, Finke C, Mannarelli C, Belachew AA, Pancrazzi A, Wassie EA, Ketterling RP et al (2014) CALR and ASXL1 mutations-based molecular prognostication in primary myelofibrosis: an international study of 570 patients. Leukemia 28:1494-1500. doi:10.1038/leu.2014.57

76. Tefferi A, Thiele J, Orazi A, Kvasnicka HM, Barbui T, Hanson CA, Barosi G, Verstovsek S, Birgegard G, Mesa R et al (2007) Proposals and rationale for revision of the World Health Organization diagnostic criteria for polycythemia vera, essential thrombocythemia, and primary myelofibrosis: recommendations from an ad hoc international expert panel. Blood 110:1092-1097. doi:10.1182/blood2007-04-083501

77. Tefferi A, Vardiman JW (2008) Classification and diagnosis of myeloproliferative neoplasms: the 2008 World Health Organization criteria and point-of-care diagnostic algorithms. Leukemia 22:14-22. doi:10.1038/sj.leu.2404955

78. Tenedini E, Bernardis I, Artusi V, Artuso L, Roncaglia E, Guglielmelli P, Pieri L, Bogani C, Biamonte F, Rotunno G et al (2014) Targeted cancer exome sequencing reveals recurrent mutations in myeloproliferative neoplasms. Leukemia 28:1052-1059. doi:10.1038/leu.2013.302

79. Teofili L, Foa R, Giona F, Larocca LM (2008) Childhood polycythemia vera and essential thrombocythemia: does their pathogenesis overlap with that of adult patients? Haematologica 93:169-172. doi:10.3324/haematol.12002

80. Teofili L, Giona F, Martini M, Cenci T, Guidi F, Torti L, Palumbo G, Amendola A, Foa R, Larocca LM (2007) Markers of myeloproliferative diseases in childhood polycythemia vera and essential thrombocythemia. J Clin Oncol 25:1048-1053. doi:10.1200/JCO. 2006.08.6884

81. Teofili L, Giona F, Martini M, Cenci T, Guidi F, Torti L, Palumbo G, Amendola A, Leone G, Foa R et al (2007) The revised WHO diagnostic criteria for $\mathrm{Ph}$-negative myeloproliferative diseases are not appropriate for the diagnostic screening of childhood polycythemia vera and essential thrombocythemia. Blood 110:33843386. doi:10.1182/blood-2007-06-094276

82. Teofili L, Giona F, Torti L, Cenci T, Ricerca BM, Rumi C, Nunes V, Foa R, Leone G, Martini M et al (2010) Hereditary thrombocytosis caused by MPLSer505Asn is associated with a high thrombotic risk, splenomegaly and progression to bone marrow fibrosis. Haematologica 95:65-70. doi:10.3324/haematol.2009.007542

83. Thiele J, Kvasnicka HM, Facchetti F, Franco V, van der Walt J, Orazi A (2005) European consensus on grading bone marrow fibrosis and assessment of cellularity. Haematologica 90:1128-1132

84. Thiele J, Kvasnicka HM, Mullauer L, Buxhofer-Ausch V, Gisslinger B, Gisslinger H (2011) Essential thrombocythemia versus early primary myelofibrosis: a multicenter study to validate the WHO classification. Blood 117:5710-5718. doi:10.1182/blood2010-07-293761

85. Vannucchi AM, Lasho TL, Guglielmelli P, Biamonte F, Pardanani A, Pereira A, Finke C, Score J, Gangat N, Mannarelli C et al (2013) Mutations and prognosis in primary myelofibrosis. Leukemia 27: 1861-1869. doi:10.1038/leu.2013.119

86. Vardiman JW, Harris NL, Brunning RD (2002) The World Health Organization (WHO) classification of the myeloid neoplasms. Blood 100:2292-2302. doi:10.1182/blood-2002-04-1199 
87. Vergara-Lluri ME, Piatek CI, Pullarkat V, Siddiqi IN, O'Connell C, Feinstein DI, Brynes RK (2014) Autoimmune myelofibrosis: an update on morphologic features in 29 cases and review of the literature. Hum Pathol 45:2183-2191. doi:10.1016/j.humpath.2014.07.017

88. Vilboux T, Lev A, Malicdan MC, Simon AJ, Jarvinen P, Racek T, Puchalka J, Sood R, Carrington B, Bishop K et al (2013) A congenital neutrophil defect syndrome associated with mutations in VPS45. N Engl J Med 369:54-65. doi:10.1056/NEJMoa1301296

89. Wilkins BS, Erber WN, Bareford D, Buck G, Wheatley K, East CL, Paul B, Harrison CN, Green AR, Campbell PJ (2008) Bone marrow pathology in essential thrombocythemia: interobserver reliability and utility for identifying disease subtypes. Blood 111:60-70. doi: 10.1182/blood-2007-05-091850

90. Wolanskyj AP, Schwager SM, McClure RF, Larson DR, Tefferi A (2006) Essential thrombocythemia beyond the first decade: life expectancy, long-term complication rates, and prognostic factors. Mayo Clin Proc 81:159-166. doi:10.4065/81.2.159

91. Yetgin S, Ozsoylu S, Ruacan S, Tekinalp G, Sarialioglu F (1989) Vitamin D-deficiency rickets and myelofibrosis. J Pediatr 114:213-217 